\title{
Embodiment and Embodied Cognition
}

\author{
Mark R. Costa ${ }^{1}$, Sung Yeun Kim ${ }^{2}$, and Frank Biocca ${ }^{2}$ \\ ${ }^{1}$ School of Information Studies, \\ ${ }^{2}$ S.I. Newhouse School of Public Communications, \\ Syracuse University M.I.N.D. Lab. \\ \{mrcosta, skim154, fbiocca\} asyr. edu
}

\begin{abstract}
Progressive embodiment and the subsequent enhancement of presence have been important goals of VR researchers and designers for some time (Biocca, 1997). Consequently, researchers frequently explore the relationship between increasing embodiment and presence yet rarely emphasize the ties between their work and other work on embodiment. More specifically, we argue that experiments manipulating or implementing visual scale, avatar customization, sensory enrichment, and haptic feedback, to name a few examples, all have embodiment as their independent variable. However, very few studies explicitly frame their work as an exploration of embodiment. In this paper we will leverage the field of Embodied Cognition to help clarify the concept of embodiment.
\end{abstract}

Keywords: human-computer interaction, presence, embodied cognition, virtual reality.

\section{Introduction}

Increasing presence is one the primary goals of virtual reality (VR) researchers and developers, whether it is intended to improve the entertainment value of the experience or ability to affect change in the user's real world behavior. A significant portion of the research and development into improving presence is centered on sensory stimulation, avatar mobility, and avatar representativeness. In the past researchers argued that these affordances all fall under embodiment (Biocca, 1997), but are in practice are rarely treated as related ideas. Despite there being a seemingly visible relationship between these research areas as well as a tentative label, very little effort has been put into clearly defining the concept linking these loosely tied areas together.

In order to resolve this problem we put forth the concept of afforded embodiment, or the degree to which the avatar provides equal or greater functionality than the user's natural body. We draw on recent developments in the area of embodied cognition to support an argument that VR researchers need to pay much closer attention to the relationship between avatar functionality and the activities within the virtual environment. The foundation of this argument is based on the basic premise of embodied cognition, which is that the body plays a constituent, not causal role in cognition (Shapiro, 2011). From this perspective, avatars with limited functionality limit their user's ability to mentally explore the virtual environment. We argue that a clearer 
understanding of the role of the body in cognition will allow researchers and designers to better prioritize design considerations for avatar functionality based on the activities or goals of the VR experience.

\section{$2 \quad$ Background and Literature Review}

\subsection{Embodied Cognition}

Embodied cognition (EC) is a research program that re-focuses cognitive science research to include the body as a critical component of cognition (Shaprio, 2007). EC assumes that our reality is shaped by the interactions of our mind, body, and environment. This contrasts with those who would argue that reality is shaped by our mind and the mind manipulates the body through abstract symbols (Shapiro, 2007). While EC is not a predictive theory, it gives virtual reality (VR) researchers an opportunity to revisit and clarify a neglected concept within our field as well as its relationship to presence.

There are two types of experimental evidence for embodied cognition - behavioral and neurological. We present the neurological evidence first and interpret the findings based on the assumption that objects and movements that activate the same neuronal systems are "linked" by the brain. For example, when examining neurons responsible for manipulating objects, objects of similar size and shape will activate the same set of neurons for a specific set of hand motions even when the subject is not actually manipulating the object, while objects of different shapes and sizes will activate a different set of neurons, even when the actual movements to manipulate the object are the same. In a subsequent study, a set of inferior premotor neurons were found to be responsible for executing a limited set of distal arm movements. However, that same set of neurons will activate if an observance within the environment is related to the physical process overseen by the neural network, even if there is no motor activity present (Gazzola \& Keysers, 2009; Pellegrino et al, 1992). The cognitive representation of the object is intrinsically tied to the way the subject uses its body to manipulate the object. They are not two separate symbols pieced together by the mind for action, but are bound together in the same schemata (Rizzolatti et al., 1988).

The behavioral examples are more straightforward and demonstrate a clear link between either sensory perception and cognitive perception or motor action and cognitive perception. For example, researchers found that by priming individuals with a warm or cold beverage they could influence whether or not the subject perceived those they just met as having a warm or cold personality, respectively (Williams \& Bargh, 2008). In an unrelated study, researchers found that sitting upright influences the extent to which subjects felt pride in an achievement. A second study found that contraction of the forehead muscles influenced subjects' perceptions of how hard they worked on a task (Stepper \& Strack, 1993).

There is also a demonstrated link between body movements and improved problem solving performance. Subjects who used appropriate body motions during a physics problem solving session performed significantly better than subjects who used inappropriate body motions (Thomas \& Lleras, 2009). In another study, researchers found that preventing the facial expression aligned to the sentiment of a sentence significantly impacted the subjects' reading performance (Havas et al., 2010). 
The previous examples reveal the extent to which the body, mind, cognitive, and emotional states are all intertwined. We create mental schemata of environmental objects via the way in which we manipulate them (Pellegrino et al., 1992; Rizzolatti et al., 1988) and encode our own bodily movements and perceive others' movements using the same set of neurons (Gazzola \& Keysers, 2009). Behaviorally, our body positioning and use impacts our social observations (Williams \& Bargh, 2008), feedback acceptance and task performance recall (Stepper \& Strack, 1993), and improves performance on cognitive tasks (Havas et al., 2010; Thomas \& Lleras, 2009). Consequently, it is critically important for VR designers and researchers to acknowledge the many subtle, yet fundamental, ways in which our body influences our perceptions, actions, and emotions.

\subsection{Embodiment}

Embodiment is a concept used in many different research areas and thus has many different, yet related meanings. In this section we will briefly discuss the different meanings and uses of embodiment, identify which of the variations we chose to adopt for this research, how we operationalize the concept, and our justifications for doing so.

Damasio (1994) and other embodied cognition theorists argue that the body serves as the central framework for our interactions with the world. We perceive the physical world in relation to our body, and therefore what we know about the world is "constructed from patterns of energy detected by the body" (Biocca, 1997). The body is also a communication device and is a critical tool for expressing mental states (Benthall \& Polhemus, 1975). The implications of this theory for VRE researchers and designers is that in order for the user to incorporate the VE into his/her reality, the system must provide affordances for users so that they may become embodied, or take some form or shape within the VRE.

Ziemcke (2003) identified six different uses of the term embodiment across multiple streams of research. Of the six notions of embodiment addressed by Ziemcke, structural coupling, historical coupling, and social embodiment are the most relevant.

Structural coupling is the notion that organisms are embodied in their environment if actions by one affect the other. Quick and colleagues (Quick, 1999 as cited in Ziemke, 2003) articulate this idea clearly, saying that "A system X is embodied in an environment $E$ if perturbatory channels exist between the two." More concretely, we can say a user is embodied in a virtual reality environment (VRE) if changes in the VRE affect the user, and the user can affect the VRE. Historical structural coupling is an extension of the idea of structural coupling in that it argues embodiment increases through a series of interactions between the system and the environment.

Ziemcke (2003) viewed social presence as orthogonal to the other definitions of presence because it addresses the role of embodiment in social situations, versus what kind of body is required for different situations. Barsalou et al. (2003) describe social embodiment as "states of the body, such as postures, arm movements, and facial expressions, [which] arise during social interaction and play central roles in social information processing (Ziemke, 2003)." Mennecke and colleagues (2010) formally incorporate this idea into their Embodied Social Presence Theory, arguing that the body is the nexus of communication and that embodied representations combined 
with goal-directed shared activities affects the perceptions of users by drawing them into higher levels of cognitive engagement.

In contrast to Ziemke's 6 different uses of embodiment, Lakoff and Johnson (1999) identify 3 distinct levels of embodiment - neural, cognitive unconscious, and phenomenological conscious experience.

Biocca's (1997) work on embodiment in VRE draws on Damasio's (1994) ideas of how the human brain constructs reality through interactions of the body with the environment, and fits within the notions of structural coupling identified by Ziemcke (2003).

While Biocca provides no formal definition of embodiment, it is clear that he views progressive embodiment as providing increasingly "natural" functionality to avatars. Natural functionality includes sensory perception in the form of high fidelity audio or visual stimulation, haptic feedback, natural motion control etc. It is also important to note that our bodies fill an important role in social interaction and selfidentification, and therefore must provide increasing affordances for how we expect to use our bodies in those situations as well.

Embodiment is expected to have a direct impact on various forms of presence (Biocca, 1997). With increasing embodiment we expect increasing levels of psychophysiological responses to VE. The brain's relationship to the body is highly malleable; therefore it is possible to convince the brain that it will suffer the consequences of actions within the VE. Biocca (1997) addresses this idea in his three-way relationships between our brain's mental models of our physical, virtual, and phenomenal selves. A second, longer-term implication of increasing embodiment - it may have a permanent effect on our body schema. We may have difficulty controlling what crosses over from virtual reality to natural reality.

Once again, it is important to note that the avatar plays a critical role in the social aspects of VE. For example, Yee and Bailenson (2007) found that users who were given taller avatars were more likely to negotiate from a position of power in online trading tasks, while those given shorter avatars were more likely to accept asymmetrical trades. Taylor (2010) provides a more descriptive account of the various ways in which users construct their identity through avatar customization, as well as the degree to which most users expect exclusive use of that identity. He also describes the process of how the user identifies with the avatar, and how that identification shapes his/her perception of self. This is a known phenomenon and has been exploited to encourage changes in real-world behavior (Dean et al., 2009).

\subsection{Neural Embodiment}

The purpose of increasing embodiment is to improve the user's sense of presence. Presence is primarily measured through a subjective post hoc questionnaire, although more recent research includes physiological measurements (Guger et al., 2004; Wiederhold et al., 2002). Further advances in psycho-physiological sensors allow us to start looking at the physiological and neurological correlates of embodiment and presence.

Within cognitive neuroscience, embodiment defined as feeling situated in one's own body (Arzy et al., 2006). This is usually researched by exploring the opposite condition, which is the out-of-body experience, or disembodiment. The most direct 
translation to VR research from cognitive neuroscience would be that a user feels as if they inhabited the avatar, with concomitant physiological responses to the environment and little notice of their own "real" body.

The results from several experiments highlight the potential for better understanding the neuro-cognitive basis for the relationship between cognitive embodiment and presence. Arzy and colleagues (2006) identified two separate regions of the brain that activated dependent upon whether subjects viewed a picture of a body from an embodied perspective, or a disembodied perspective. The former condition resulted in activation of the extrastriate body area (EBA), while the latter resulted in the tempoparietal junction (TPJ). Injury to the TPJ has been linked to out-of-body experiences in other research (Blanke et al, 2004), and has been associated with certain aspects of self-processing, self-other distinction, and mental own-body imagery (Arzy et al., 2006; Ruby \& Decety, 2001; Vogeley \& Fink, 2003). The EBA responds to both images of bodies and body parts, imagined movement of one's own body, and executed movements (Arzy et al., 2006; Astafiev et al., 2004; Downing et al., 2001).

A similar result was found when researchers asked subjects to imagine themselves at some location outside of their body and then perform spatial transformations on the body (Blanke and Arzy, 2005). Subjects were then asked to perform the same task with nonbody images. Subsequent artificially induced interference through transcranial magnetic stimulation interfered with the former task, but not the latter. The results suggest that the TPJ is responsible for mediating spatial unity of self and body, not and that external representations of self are not treated as normal objects for spatial transformation.

Research in cognitive neuroscience on embodiment is relevant to our explorations of embodiment and presence in virtual worlds. Understanding which regions of the brain are responsible for creating the feeling of inhabiting an avatar will allow us to better measure the user's reaction to the avatar.

\section{Afforded Embodiment and Virtual Reality}

Taking these ideas and aspects of embodiment, we define embodiment as the degree to which an avatar affords the user equal or greater functionality expected of our natural bodies. This functionality is comprised of three dimensions - physical [motor control and environmental manipulation], sensory input, social and self-identity. We argue that researchers have been experimenting with different degrees of afforded embodiment for years yet have not really considered their research to be part of embodiment research.

Our dimensions of embodiment map well to Lee's (K. M. Lee, 2004) three forms of presence, although the strengths of those relationships has yet to be determined. Evidence suggests that a lack of self-presence may inhibit achieving full presence (Slater, Usoh, \& Steed, 1995), which would also indicate that pyscho-social embodiment is always an important consideration.

In addition to progressive sensory and motor embodiment, there needs to be a high degree of sensorimotor coupling. This is defined as the "degree to which changes in body position correlate immediately and naturally with appropriate changes in sensory feedback (Biocca, 1997)." For example, a lack of coordination between the visual, vestibular, and motor systems usually results in simulator sickness. 


\subsection{Sensory Input}

The sensory input dimension measures the degree to which the user can leverage the different senses during interactions with the VE. These include, but are not limited to - vision, audition, olfaction, tactician, thermoception, proprioception, etc.

The body of research on visual sensory stimulation in VE is very large; this is not surprising given the amount of emphasis our culture places on the visual. This research includes experimentation on visual scale (i.e., screen size) (Tan et al., 2004) and dimensionality (2D vs. 3D) (Bae et al., 2012) and their effects on a range of task performances and presence.

Higher quality ambient and action driven sounds (i.e., sounds appearing to originate from the source of movement) are increasingly common. A number of researchers have been working on the role of audio in virtual worlds and have found varying degrees of effect on subjective reports of subjects' level of immersion (Grimshaw, Lindley, \& Nacke, 2008).

Haptic feedback is an increasingly popular affordance in virtual environments. Force feedback has been a popular option in many simulators, and is now relatively common on many joysticks. More sophisticated implementations are also popular, as researchers seek to increase users' sense of presence. Sallnäs and colleagues (Sallnäs, Rassmus-Gröhn, \& Sjöström, 2000) found that implementing a force feedback mechanism had positive effects on [physical] presence and task performance. There have also been a number of successful subsequent studies looking to use haptic feedback for improving or understanding social (Bailenson \& Yee, 2007; Chan, MacLean, \& McGrenere, 2008) and physical (S. Lee \& Kim, 2008) presence in virtual environments.

\subsection{Motor Control}

Motor control refers to the ways in which the user can control the avatar as well as the degree to which the avatar is controllable. Avatar control generally refers to the input device used to control the movements of the avatar (gamepad, keyboard, etc.). Avatars can move through VE via different paths; in early video games movement was along one or two dimensions, while newer VEs allow much greater freedom of movement. The ability to, and activity of, engaging in body movement including bending, crouching, and head pitch and yaw, affects presence (Slater et al, 1998). Researchers have also found that mapping VE locomotion to similar real body movements results in higher presence, albeit mediated by the amount of subjective association the user has with the avatar (Slater et al., 1995). Finally, users can manipulate the VE to varying extents; how the user manipulates the environment as well as the degree to which she can has an effect on presence.

\subsection{Psycho-social Afforded Embodiment}

Psycho-social afforded embodiment refers to the degree to which the user can modify and/or manipulate their avatar to reflect or express their identity. That identity can either be an idealized or accurate reflection of their identity. The avatar must facilitate, or at least not impede, the process of identity construction. Based on previous research we know that avatar customization is an important affordance and that users 
expend considerable effort to customize their avatar (Ducheneaut et al, 2009; Taylor, 2002), respond and behave according to the embodiment of their avatar (Yee \& Bailenson, 2007), and feel higher levels of presence when the avatars resemble themselves (Bailey, Wise, \& Bolls, 2009). Psycho-social embodiment directly affects selfpresence and social presence, and may serve as a moderating variable for physical presence.

\section{Conclusions}

In this paper we discussed the ways in which the interactions between our minds, bodies, and environment form the basis for our cognition. For example, we create mental schemata of environmental objects via the way in which we manipulate them (Pellegrino et al., 1992; Rizzolatti et al., 1988). We also encode our own bodily movements and perceive others' movements using the same set of neurons (Gazzola \& Keysers, 2009). Behaviorally, our body positioning and use impacts our social observations (Williams \& Bargh, 2008), feedback acceptance and task performance recall (Stepper \& Strack, 1993), and improves performance on cognitive tasks (Havas et al., 2010; Thomas \& Lleras, 2009).

The three-way relationship between mind, body and environment is the focus of the research area known as Embodied Cognition, which in turn can be leverage to guide VR researchers and designers who are interested in making VR systems that facilitate greater levels of presence. More importantly, by acknowledging that our bodies play an integral (instead of subordinate) role in our cognitive processes and that this role has multiple dimensions we can begin to explore the more subtle yet important relationships between embodiment and presence.

Looking to the future we see opportunities to not only revisit old data, but also start exploring the ways in which avatar design affordances affects users' sense of presence in virtual environments. Additionally, we now have a framework that can be used to guide psycho-physiological instrument based research on presence. For example, it may be possible to use functional near infrared spectroscopy (fNIRS) and electroencephalography (EEG)(Hirshfield et al., 2009) to measure users' engagement, mental workload, and response inhibitions in virtual environments to see if the affordances are working as intended.

Based on the ideas of embodied cognition, we argued that afforded embodiment is an appropriate framework for exploring avatar functionality and presence. In this paper we highlighted the already large body of literature built up around exploring degrees of embodiment. While that research is a good first step, much of it did not seek to explicitly measure the relationships between the dimensions of afforded embodiment and forms of presence. Insight into these relationships will help designers and researchers make more informed design decisions when choosing avatar affordances for virtual environments. Finally, establishing a more systematic and coherent view of the relationship between the user and their avatar is a necessary first step in understanding the user's experience in virtual environments. 
Acknowledgement. This work was supported in part by grant No. R31-10062 from the World Class University (WCU) project of the Korean Ministry of Education, Science \& Technology (MEST) and the Korea National Research Foundation (NRF) through Sungkyunkwan University (SKKU Univ.). The authors sincerely thank MEST and NRF for providing valuable resources to this research project. The project was also supported in part by the Newhouse endowment awarded to Frank Biocca.

\section{References}

1. Arzy, S., Thut, G., Mohr, C., Michel, C.M., Blanke, O.: Neural basis of embodiment: distinct contributions of temporoparietal junction and extrastriate body area. The Journal of Neuroscience the Official Journal of the Society for Neuroscience 26(31), 8074-8081 (2006), doi:10.1523/JNEUROSCI.0745-06.2006

2. Astafiev, S.V., Stanley, C.M., Shulman, G.L., Corbetta, M.: Extrastriate body area in human occipital cortex responds to the performance of motor actions. Nature neuroscience 7(5), 542-548 (2004), doi:10.1038/nn1241

3. Bae, S., Lee, H., Park, H., Cho, H., Park, J., Kim, J.: The effects of egocentric and allocentric representations on presence and perceived realism: Tested in stereoscopic 3D games. Interacting with Computers 24(4), 251-264 (2012), doi:10.1016/j.intcom.2012.04.009

4. Bailenson, J.N., Yee, N.: Virtual interpersonal touch: Haptic interaction and copresence in collaborative virtual environments. Multimedia Tools and Applications 37(1), 5-14 (2007), doi:10.1007/s11042-007-0171-2

5. Bailey, R., Wise, K., Bolls, P.: How avatar customizability affects children's arousal and subjective presence during junk food-sponsored online video games. Cyberpsychology \& Behavior: The Impact of the Internet, Multimedia and Virtual Reality on Behavior and Society 12(3), 277-283 (2009), doi:10.1089/cpb.2008.0292

6. Biocca, F.: The cyborg's dilemma: embodiment in virtual environments. In: Proceedings Second International Conference on Cognitive Technology Humanizing the Information Age, pp. 12-26. IEEE Comput. Soc (1997), doi:10.1109/CT.1997.617676

7. Blanke, O., Landis, T., Spinelli, L., Seeck, M.: Out-of-body experience and autoscopy of neurological origin. Brain: A Journal of Neurology 127(Pt. 2), 243-258 (2004), doi:10.1093/brain/awh040

8. Chan, A., MacLean, K., McGrenere, J.: Designing haptic icons to support collaborative turn-taking. International Journal of Human-Computer Studies 66(5), 333-355 (2008), doi:10.1016/j.ijhes.2007.11.002

9. Damasio, A.R.: Descartes' Error: Emotion, reason, and the brain, p. 312. Putname, New York (1994)

10. Dean, E., Cook, S., Keating, M., Murphy, J.: Does this Avatar Make Me Look Fat? Obesity and Interviewing in Second Life. Journal of Virtual Worlds Research 2(2) (2009), doi:10.4101/jvwr.v2i2.621

11. Decety, J., Grezes, J.: Neural mechanisms subserving the perception of human actions. Trends in Cognitive Sciences 3(5), 172-178 (1999), doi:10.1016/S1364-6613(99)01312-1

Ducheneaut, N., Wen, M.-H., Yee, N., Wadley, G.: Body and mind. In: Proceedings of the 27th International Conference on Human Factors in Computing Systems - CHI 2009, p. 1151. ACM Press, New York (2009), http://dl.acm.org.libezproxy2. syr.edu/citation. cfm?id=1518701.1518877 
12. Gazzola, V., Keysers, C.: The observation and execution of actions share motor and somatosensory voxels in all tested subjects: single-subject analyses of unsmoothed fMRI data. Cerebral cortex 19(6), 1239-1255 (1991), doi:10.1093/cercor/bhn181

13. Gibbs, R.W.: Metaphor Interpretation as Embodied Simulation. Mind \& Language 21(3), 434-458 (2006), doi:10.1111/j.1468-0017.2006.00285.x

14. Grimshaw, M., Lindley, C., Nacke, L.: Sound and Immersion in the First-Person Shooter: Mixed Measurement of the Player's Sonic Experience. Scientific Commons, 7 (2008), http://en.scientificcommons.org/49379075 (retrieved)

15. Guger, C., Edlinger, G., Leeb, R., Pfurtscheller, G., Antley, A., Garau, M., Brogni, A., et al.: Heart-Rate Variability and Event-Related ECG in Virtual Environments. In: 7th Annual International Workshop on Presence, Valencia, Spain, pp. 240-245 (2004),

http: / / citeseerx. ist.psu.edu/viewdoc/summary?doi=10.1.1.127. 361 (retrieved)

16. Havas, D.A., Glenberg, A.M., Gutowski, K.A., Lucarelli, M.J., Davidson, R.J.: Cosmetic use of botulinum toxin-a affects processing of emotional language. Psychological Science 21(7), 895-900 (2010), doi:10.1177/0956797610374742

17. Hirshfield, L.M., Chauncey, K., Gulotta, R., Girouard, A., Solovey, E.T., Jacob, R.J.K., Sassaroli, A., Fantini, S.: Combining Electroencephalograph and Functional Near Infrared Spectroscopy to Explore Users' Mental Workload. In: Schmorrow, D.D., Estabrooke, I.V., Grootjen, M. (eds.) FAC 2009. LNCS, vol. 5638, pp. 239-247. Springer, Heidelberg (2009), doi:10.1007/978-3-642-02812-0

18. Lakoff, G., Johnson, M.: Philosophy in the flesh: The embodied mind and its challenge to Western thought, p. 640. Basic Books, New York (1999),

http: //books.google.com/books?hl=en\&lr=\&id=KbqxnX3_uc0C\&pgis=1

19. Lee, K.M.: Presence, Explicated. Communication Theory 14(1), 27-50 (2004), doi:10.1111/j.1468-2885.2004.tb00302.x

20. Lee, S., Kim, G.J.: Effects of haptic feedback, stereoscopy, and image resolution on performance and presence in remote navigation. International Journal of Human-Computer Studies 66(10), 701-717 (2008), doi:10.1016/j.ijhcs.2008.05.001

21. Mennecke, B.E., Triplett, J.L., Hassall, L.M., Conde, Z.J.: Embodied Social Presence Theory. In: 2010 43rd Hawaii International Conference on System Sciences, pp. 1-10. IEEE (2010), doi:10.1109/HICSS.2010.179

22. Pellegrino, G., Fadiga, L., Fogassi, L., Gallese, V., Rizzolatti, G.: Understanding motor events: a neurophysiological study. Experimental Brain Research 91(1) (1992), doi:10.1007/BF00230027

23. Quick, T., Dautenhahn, K.: On Bots and Bacteria: Ontology Independent Embodiment. In: Floreano, D., Mondada, F. (eds.) ECAL 1999. LNCS, vol. 1674, pp. 339-353. Springer, Heidelberg (1999),

http: / / citeseer.ist.psu.edu/viewdoc/summary?doi=10.1.1.17.9469

24. Rizzolatti, G., Camarda, R., Fogassi, L., Gentilucci, M., Luppino, G., Matelli, M.: Functional organization of inferior area 6 in the macaque monkey. Experimental Brain Research 71(3), 491-507 (1988), doi:10.1007/BF00248742

25. Ruby, P., Decety, J.: Effect of subjective perspective taking during simulation of action: a PET investigation of agency. Nature neuroscience 4(5), 546-550 (2001), doi:10.1038/87510

26. Sallnäs, E.-L., Rassmus-Gröhn, K., Sjöström, C.: Supporting presence in collaborative environments by haptic force feedback. ACM Transactions on Computer-Human Interaction 7(4), 461-476 (2000), doi:10.1145/365058.365086 
27. Shapiro, L.A.: Embodied Cognition (Google eBook), p. 237. Taylor \& Francis (2011), http: / /books.google. com/books?hl=en\&lr=\&id=Msi 50Zek9vwC\&pgis=1 (retrieved)

28. Slater, M., Steed, A., McCarthy, J., Maringelli, F.: The Influence of Body Movement on Subjective Presence in Virtual Environments. Human Factors: The Journal of the Human Factors and Ergonomics Society 40(3), 469-477 (1998), doi:10.1518/001872098779591368

29. Slater, M., Usoh, M., Steed, A.: Taking steps: the influence of a walking technique on presence in virtual reality. ACM Transactions on Computer-Human Interaction 2(3), 201-219 (1995), doi:10.1145/210079.210084

30. Smith, L.B.: Cognition as a dynamic system: Principles from embodiment. Developmental Review 25(3-4), 278-298 (2005), doi:10.1016/j.dr.2005.11.001

31. Stepper, S., Strack, F.: Proprioceptive determinants of emotional and nonemotional feelings. Journal of Personality and Social Psychology 64(2), 211-220 (1993)

32. Tan, D.S., Gergle, D., Scupelli, P.G., Pausch, R.: Physically large displays improve path integration in 3D virtual navigation tasks. In: Proceedings of the 2004 Conference on $\mathrm{Hu}-$ man Factors in Computing Systems - CHI 2004, pp. 439-446. ACM Press, New York (2004), doi:10.1145/985692.985748

33. Taylor, T.L.: Living digitally: Embodiment in virtual worlds. In: Schroeder, R. (ed.) The social life of avatars: presence and interaction in shared virtual environments, pp. 40-62. Springer, London (2002)

34. Thomas, L.E., Lleras, A.: Swinging into thought: directed movement guides insight in problem solving. Psychonomic bulletin \& review 16(4), 719-723 (2009), doi:10.3758/PBR.16.4.719

35. Vogeley, K., Fink, G.R.: Neural correlates of the first-person-perspective. Trends in cognitive sciences 7(1), 38-42 (2003), http: / /www.ncbi.nlm.nih.gov/pubmed/12517357 (retrieved)

36. Wiederhold, B.K., Jang, D.P., Kim, S.I., Wiederhold, M.D.: Physiological Monitoring as an Objective Tool in Virtual Reality Therapy. CyberPsychology \& Behavior 5(1), 77-82 (2002), doi:10.1089/109493102753685908

37. Williams, L.E., Bargh, J.A.: Experiencing physical warmth promotes interpersonal warmth. Science 322(5901), 606-607 (2008), doi:10.1126/science.1162548

38. Yee, N., Bailenson, J.: The Proteus Effect: The Effect of Transformed Self-Representation on Behavior. Human Communication Research 33(3), 271-290 (2007), http://doi.wiley.com/10.1111/j.1468-2958.2007.00299.x (retrieved)

39. Ziemke, T.: What's that thing called embodiment. In: Proceedings of the 25th Annual meeting of the Cognitive Science Society, pp. 1305-1310 (2003) 